Archives

16 | 1996

Pour une histoire comparée du vœu

\title{
Angelo Clareno et le vœu d'obéissance
}

\section{David Burr}

\section{OpenEdition \\ Journals}

\section{Édition électronique}

URL : http://journals.openedition.org/ccrh/2643

DOI : $10.4000 /$ ccrh. 2643

ISSN : 1760-7906

\section{Éditeur}

Centre de recherches historiques - EHESS

\section{Édition imprimée}

Date de publication : 16 avril 1996

ISSN : 0990-9141

\section{Référence électronique}

David Burr, "Angelo Clareno et le vœu d'obéissance », Les Cahiers du Centre de Recherches Historiques [En ligne], 16 | 1996, mis en ligne le 27 février 2009, consulté le 20 avril 2019. URL : http:// journals.openedition.org/ccrh/2643; DOI : 10.4000/ccrh.2643

Ce document a été généré automatiquement le 20 avril 2019.

Article L.111-1 du Code de la propriété intellectuelle. 


\title{
Angelo Clareno et le vœu d'obéissance
}

\author{
David Burr
}

1 En 1317, détenu en Avignon, Angelo Clareno a écrit son Epistola excusatoria au pape Jean XXII. Dans cette lettre, Angelo répond à l'accusation d'avoir désobéi à une bulle du pape Boniface VIII. Il explique l'origine de son groupe, les pauvres ermites du pape Célestin, et il insiste sur le fait que le pape Célestin leur avait commandé d'obéir à la règle et au testament de saint François. Quant aux supérieurs humains, Célestin leur avait dit: "Je veux que vous ne soyez obligés d'obéir qu'à moi, et à frère Liberato, votre chef. » Autrement dit, il les avait établis en tant qu'ordre distinct.

2 Comme presque tous les franciscains spirituels de cette époque, Angelo présente son argument comme s'il s'agissait d'une question d'obéissance. Il a fait son vœu, puis le pape lui a donné un ordre particulier. Il veut y obéir. Mais il ne peut pas obéir si Jean ne reconnaît pas la légitimité de l'Ordre des pauvres ermites. Le raisonnement d'Angelo peut sembler bizarre, voire malhonnête. Il veut obéir au pape Célestin, mais aussi ignorer les instructions du pape Boniface VIII qui avait choisi, lui, de considérer Angelo et son groupe comme membre de l'ordre franciscain et non comme ordre séparé. Boniface ne voulait pas reconnaître les pauvres ermites comme ordre légitime.

3 L'argumentation est typique. Entre 1313 et 1317, dans leurs écrits polémiques, les franciscains spirituels de Narbonne et de Béziers ne voulaient pas trop accentuer les exigences du vœu de pauvreté. Ils ont préféré parler du vœu d'obéissance en partie parce que la bulle de Clément V, Exivi de paradiso (1312) avait interdit la continuation du débat sur la question de l'usus pauper. C'était aussi que la question centrale, pour les deux factions, n'était plus celle de l'usus pauper, mais celle de savoir quelle faction, des spirituels ou de leurs chefs, obéissait à la tentative de Clément pour résoudre la controverse entre elles. Dans le Midi, les spirituels s'étaient emparés par la violence des maisons franciscaines de Béziers et de Narbonne, et ces deux maisons étaient devenues des refuges où d'autres spirituels avaient pu trouver asile. Les chefs raisonnaient ainsi : Clément avait commandé aux spirituels d'obéir à leurs supérieurs et les spirituels désobÈissaient à cet ordre. Les spirituels insistaient sur le fait que leur rébellion contre 
leurs chefs provinciaux était nécessaire précisément parce que ces chefs s'étaient euxmêmes rebellés contre le règlement de Clément, en réintégrant dans le sud de la France des dirigeants qui étaient très hostiles aux spirituels, et en refusant de permettre aux trois maisons du sud de la France - Narbonne, Béziers et Carcassonne - de continuer d'être des îlots d'observance spirituelle. Ils s'étaient donc rebellés contre une autorité inférieure relativement à l'obéissance à l'autorité papale. Ils disaient aussi que leur rébellion était nécessaire parce que, comme franciscains, ils avaient fait vœu d'observer la règle, mais la littérature polémique existante suggère qu'ils voyaient les périls de ce deuxième argument. Ils considéraient la loyauté au pape comme un argument moins dangereux que la loyauté à la règle.

4 Jean a donné sa réponse aux spirituels en 1317. Ce fut la bulle Quorumdam exigit, qui les décrit comme des schismatiques dont le scrupule abusif les mène à refuser l'obéissance à leurs supérieurs légitimes. Jean leur avait commandé de cesser leur rébellion et d'obéir à leurs chefs. Une fois que Jean eut fait venir à Avignon les rebelles de Narbonne et de Béziers, et quand les supérieurs franciscains leur eurent demandé s'ils obéiraient à la décision du pape, toute discussion du règlement clémentin était devenue inutile. S'ils décidaient de défier Jean, ils ne pouvaient justifier ce défi qu'en insistant sur le fait que leur loyauté fondamentale était due à leur vœu, non au pape.

Et c'est précisément la manière dont ceux qui ont refusé d'obéir ont expliqué leur action. C'était une justification très olivienne. Ils avaient hérité de Pierre de Jean Olivi une théorie de la pauvreté qui justifiait (et, en fait, demandait) la désobéissance quand un supérieur-qu'il fût gardien local, ministre provincial, ministre général ou pape - ordonnait la violation de l'usus pauper. Ils devaient ainsi fuir ou mourir. D'ailleurs, plusieurs avaient également hérité d'olivi une théologie de l'histoire qui clarifiait le comportement de ces mêmes supérieurs. Quand on comprenait les objectifs de l'Église charnelle et de son chef, l'Antéchrist, ce comportement devenait clairement explicable. Cette dimension apocalyptique n'avait pas échappé à l'attention de Jean, comme nous le voyons dans la bulle Gloriosam Ecclesiam.

6 Après la décision de Jean, Angelo, comme les spirituels français, dut choisir entre l'obéissance et la résistance. Il ne choisit ni l'une ni l'autre. Ou peut-être pourrait-on dire qu'il choisit les deux. Il n'a pas dû affronter la fureur des supérieurs franciscains, parce que le pape lui a donné la permission d'entrer dans un autre ordre, celui des célestins. Mais il a continué de conseiller beaucoup de franciscains spirituels italiens. En 1318, il est revenu en Italie, où il a passé le reste de la vie, et où il est devenu le chef de plusieurs spirituels.

7 Mais, avant même de quitter Avignon, Angelo avait donné à ses collègues italiens d'importants conseils. Dans une lettre écrite probablement en 1317, il explique qu'il y a des gens qui croient posséder la réalité quand ils n'en possèdent que le nom. Ils croient que, s'ils ont les maisons, les vêtements, et les supérieurs, ils ont l'ordre créé par saint François. Mais ces bâtiments, ces vêtements, ces offices n'existent que pour faciliter l'observance de la règle. Sans cette observance, on n'a pas la réalité. L'obéissance à la règle est plus importante que l'obéissance aux supérieurs. La règle peut avoir sa réalité et ses observateurs dévots, même quand le nom de l'ordre, ses murs, ses toits, ses cérémonies externes disparaissent. Quant à Angelo, saint François a prophétisé que les supérieurs de l'ordre choisiraient le nom et non pas la réalité. Il a donc préféré parler, non de l'ordo minorum, mais de la vita minorum. 
Quelques années plus tard, en 1328, quand Philippe de Majorque cherche à obtenir de Jean XXII l'autorisation de créer un nouvel ordre religieux dans le but d'observer la règle de saint François dans sa pureté, Angelo écrit à Philippe, et il lui donne un conseil. « Je ne trouve, dit-il, aucun disciple du Christ qui ait demandé l'avis de quelque prêtre [du Temple] ou de quelque chef de la Synagogue. Je ne crois pas donc que quelqu'un qui soit sain d'esprit, après avoir reçu une illumination sûre du Saint-Esprit, puisse, sans offense, chercher des conseils sur la question de comment il doit mener sa vie, de la part de quelqu'un qui vit et enseigne selon la chair. » Et il encourage Philippe à prendre comme modèle de conduite les martyrs et les confesseurs de l'Église primitive. Une lettre de cette époque à quelques collègues italiens propose comme modèle les années $\mathrm{du} \mathrm{IV}^{\mathrm{e}}$ siècle où l'empereur et la hiérarchie ecclésiastique étaient ariens et où la vraie Église ne se trouvait qu'en peu de personnes.

9 Évidemment, Angelo croit vivre une époque où il faut compter sur la conscience individuelle. On ne doit pas trop compter sur l'autorité ecclésiastique. Évidemment, il considère le pape comme la voix de la carnalité. Le voit-il aussi comme l'Antéchrist ? C'est là une question plus difficile. Angelo est réticent sur ce sujet. Cependant, si l'on étudie bien ses paroles, il est difficile d'éviter la conclusion que, lorsqu'il cite son chapitre apocalyptique favori de l'évangile de Matthieu, chapitre 24 , verset 15 : «Lorsque vous verrez l'abomination de la désolation, dont a parlé le prophète Daniel, établie en lieu saint »-, Angelo croît bien savoir où se trouve l'abomination de la désolation. Elle est, en fait, établie en Avignon.

10 Mais les lettres d'Angelo à Philippe de Majorque et à ses collègues italiens n'encouragent jamais un témoignage agressif, ni pour la pauvreté ni contre le pape. Elles n'encouragent jamais une résistance publique. En 1330, Philippe notifia à Angelo qu'il voulait déclarer à $\mathrm{Jean}^{\dagger}$ XXII son opinion sur la pauvreté, une opinion, faut-il le dire, très différente de celle de Jean. Angelo répond à Philippe que rendre publique une telle différence d'opinion ne saurait aider leur cause. "Écrire au pape ou aux rois, dit-il, ne me semble pas une stratégie utile, parce que l'avarice, l'amour des choses visibles et le désir de régner les séparent de l'amour de la pauvreté. » L'époque contemporaine est comme celle du Christ, quand les rois, les prêtres et les savants s'unissaient tous contre lui. Dans une telle époque, on doit confesser sa foi sans réticence, mais on ne doit pas juger le pape; en fait, il n'y a aucun besoin de le juger, parce que le pape s'est jugé lui-même. Il faut se consacrer aux poursuites spirituelles modestes, et laisser tout jugement au Christ et aux chefs de l'Église. Une lettre à ses collègues italiens annonce que, si la situation particulière l'exige, il faut confesser la vérité, mais qu'autrement le comportement convenable est «le silence, la discrétion, l'isolement, et la patience ".

11 Évidemment, les conseils d'Angelo reflètent son réalisme sur les possibilités de son temps, mais ils reflètent aussi sa pensée sur les exigences de la règle et du testament de saint François. Quand la controverse entre Jean XXII et les supérieurs de l'ordre franciscain est devenue une lutte plus sérieuse, Philippe de Majorque écrit de nouveau à Angelo, et lui demande de donner son appui à un parti ou à l'autre. Angelo refuse de prendre position. La charité et l'humilité, dit-il, nous requièrent d'éviter de telles luttes. D'ailleurs, si l'on veut rester humble, on ne peut s'opposer au pape ni même le corriger. Le Christ seul peut corriger un mauvais pape.

Dans une lettre à quelques amis italiens, Angelo va encore plus loin. Le Christ a enseigné à ses disciples à « honorer les prêtres avares et illégitimes, les scribes odieux et menteurs, les pharisiens hypocrites et frauduleux, à cause de la dignité de leur état, et pour 
conserver aussi la paix et l'unité. Les chrétiens ne sont-ils donc pas encore plus obligés d'être obéissants aux chefs de l'Église, qui, même s'ils sont coupables, occupent la place du Christ et de ses apôtres?».

On commence à comprendre pourquoi Angelo propose les martyrs et les confesseurs de l'Église primitive comme modèles de conduite. Il peut sembler qu'il n'y a rien à faire sauf confesser sa foi et se soumettre à la persécution. Mais Angelo lui-même ne subit pas la persécution, et il ne semble confesser sa foi que dans les lettres à ses amis. Nous devons ainsi chercher un autre élément dans ses conseils. On le trouve dans son chapitre apocalyptique favori : «Lorsque vous verrez l'abomination de la désolation, dont a parlé le prophète Daniel, établie en un lieu saint,... alors, que ceux qui seront en Judée fuient dans les montagnes... » Selon Angelo, il ne faut pas prendre littéralement ces mots, « fuir dans les montagnes ", qui peuvent signifier confesser la vie et la doctrine de Christ dans son cœur et sa vie. Ils peuvent entraîner ou non un changement de localité. Mais Angelo lui-même pouvait prendre le conseil littéralement. Il consacre ses derniers jours à une une vie érémitique à Subiaco, puis à Santa Maria de Aspro et à San Michele dans le Basilicate. C'était une solution parfaite pour lui et ses collègues. On pouvait confesser la vie et la doctrine de Christ, mais il ne fallait pas les confesser devant les chefs de l'Église. Il n'y avait pas besoin de confrontation. On n'avait besoin que d'un évêque ou d'un abbé bienveillant. Angelo trouve ce dernier en Bartolomeo, abbé de Subiaco.

Ici, je crois que nous affrontons quelques éléments centraux de la pensée et, si l'on peut dire, de la stratégie d'Angelo. Il faut obéir au vœu, et cela signifie mener une vie de pauvreté et d'humilité. Si les supérieurs franciscains ou le pape en ordonnent autrement, on doit néanmoins obéir au vœu, même si cette obéissance mène au martyre. Mais on ne doit pas corriger ses supérieurs, on ne doit pas les assaillir, parce qu'ils occupent la place $\mathrm{du}$ Christ et de ses apôtres. Mais bien qu'on doive leur obéir, bien qu'on ne doive pas les corriger, on peut quelquefois les éviter. On peut exister et obéir au vœu sans se confronter à qui que ce soit, et sans souffrir. On peut voyager en Arménie, on peut séjourner en Grèce, on peut demeurer à Subiaco. On peut « fuir dans les montagnes ». Ce fut souvent la stratégie d'Angelo.

C'était une stratégie plus ou moins efficace. Au moment de sa mort, les inquisiteurs le surveillaient, bien sûr ; mais il mourut assez vieux

\section{AUTEUR}

\section{DAVID BURR}

Virginia Tech, Blacksburg, Va, USA 